\title{
THERMOELECTRIC POWER STUDIES ON A MARTENSITIC STAINLESS STEEL
}

\author{
F. G. Caballero, C. Capdevila, L. F. Alvarez and C. García de Andrés \\ Solid-Solid Phase Transformation Group (GITFES), Department of Physical Metallurgy, Centro \\ Nacional de Investigaciones Metalúrgicas (CENIM), Consejo Superior de Investigaciones \\ Científicas (CSIC), Avda. Gregorio del Amo, 8, 28040 Madrid, Spain, www.cenim.csic.es
}

Keywords: Stainless steels, Carbides, Thermoelectric power

\begin{abstract}
In this work the different contributions to the thermoelectric power (TEP) measurement during the dissolution process of $\mathrm{M}_{23} \mathrm{C}_{6}$ carbides in an X45Cr13 stainless steel have been evaluated. Thus, the influences of carbon and chromium contents in solid solution in the austenite, the austenite grain boundaries and the microstructure on the TEP have been determined.
\end{abstract}

\section{INTRODUCTION}

Martensitic stainless steels containing $13 \% \mathrm{Cr}$ are commonly used in quenched and tempered conditions [1-2]. Quenching heat treatment involved annealing to obtain austenite and to dissolve the carbides, followed by cooling to transform the austenite into martensite. There may also be some retained austenite present in the microstructure since carbon and chromium lower the martensite transformation temperature retaining austenite at room temperature.

The amount of carbides in the quenched microstructure exerts an important influence on the properties of these materials. Therefore, the study of the dissolution process of carbides as a function of austenitization temperature is of great importance to optimize the properties of these steels. TEP measurement is a powerful method to study the dissolution process of carbides. In fact, this technique is very sensitive to the amount of atoms in solid solution in iron [3-7]. In this work the different contributions to the TEP during the dissolution process of $\mathrm{M}_{23} \mathrm{C}_{6}$ carbides in an $\mathrm{X} 45 \mathrm{Cr} 13$ stainless steel have been evaluated. Thus, the influences of the carbon and chromium content in solid solution in the austenite, austenite grain boundaries and the microstructure on the TEP have been determined.

\section{EXPERIMENTAL PROCEDURE}

The chemical composition of the studied steel in wt-\% is as follows: $0.45 \% \mathrm{C}, 13.0 \% \mathrm{Cr}, 0.38 \% \mathrm{Ni}$, $0.32 \% \mathrm{Si}, 0.44 \% \mathrm{Mn}, 0.03 \% \mathrm{P}$, and $0.016 \% \mathrm{~S}$. The material was obtained from a cold-rolled coil and was tested in annealed condition. As-received microstructure of this steel is presented elsewhere [8]. This microstructure consists of globular carbides finely distributed in a ferrite matrix. Thermodynamic equilibrium calculations and x-ray diffraction patterns elsewhere showed only the presence of $\mathrm{M}_{23} \mathrm{C}_{6}$ carbides in the as-received and as-quenched microstructures [8-9].

In order to study the dissolution process of $\mathrm{M}_{23} \mathrm{C}_{6}$ carbides in the steel by means of TEP measurements, samples $30 \mathrm{~mm}$ in length, $2 \mathrm{~mm}$ in width and $0.8 \mathrm{~mm}$ in thickness were austenitized in vacuum at different temperatures (1010, 1060, 1106, 1120, 1168, 1200 and $\left.1232{ }^{\circ} \mathrm{C}\right)$. After 180 seconds of holding time, specimens were cooled at $50{ }^{\circ} \mathrm{C} / \mathrm{s}$. Previous work in this steel showed that this cooling rate is high enough to avoid carbide precipitation during cooling [10].

Measurements of the area percentage occupied by carbides in the microstructure were performed by means of an automatic image analyzer (Kontron IBAS2). Test pieces etched with Murakami's 
reagent were used for these measurements. Figure 1 shows the optical micrographs of the as received and heat-treated microstructures of the studied steel etched with this reagent.

The microstructure of the heat-treated samples consists, in all cases, of martensite and some retained austenite. Quantitative X-ray analysis was used to determine the volume fraction of retained austenite [11]. Vickers hardness tests were carried out on samples heat treated at different austenitization temperature. The results correspond to the average of at least five tests. The prior austenite grain size (PAGS) for the different austenitization conditions was estimated in gasquenched samples using a linear intercept procedure [12].

A schematic representation of the TEP apparatus is given elsewhere [7]. The experimental procedure of the TEP measurement is the following: the sample is pressed between two blocks of a reference metal (in this case, pure copper). One of the blocks is at $15{ }^{\circ} \mathrm{C}$, while the other is at $25{ }^{\circ} \mathrm{C}$ to obtain a temperature difference $\Delta T$. A potential difference $\Delta V$ is generated at the reference metal contacts. The apparatus does not give the absolute the TEP value of the sample $\left(S^{*}\right)$, but a relative

TEP $(S)$ in comparison to the TEP of pure copper $\left(S_{o}^{*}\right)$ at $20{ }^{\circ} \mathrm{C}$. $S$ is given by the following relation:

$$
S=S^{*}-S_{o}^{*}=\frac{\Delta V}{\Delta T}
$$

The TEP value does not depend on the shape of the sample, which is a great advantage of this technique. Moreover, the measurement is performed very quickly (less than $1 \mathrm{~min}$ ) and precisely (about $\pm 0.5 \%$ ). The resolution is of the order of $0.001 \mu \mathrm{V} / \mathrm{K}$.

\section{RESULTS AND DISCUSSION}

To follow the effects of heat treatments on a specific material, it is convenient to use a relative TEP scale with this material as the reference level. Thus, the TEP value corresponding to the lowest austenitization temperature tested $\left(1010^{\circ} \mathrm{C}\right)$, equal to $8.277 \mu \mathrm{V} / \mathrm{K}$, was chosen as the reference level for the study of carbide dissolution as a function of austenitization temperature in the steel. At this austenitizing state the majority of carbon and chromium of the steel has precipitated in form of carbides. The TEP measurements were performed on samples with a martensite plus retained austenite microstructure. As a first approximation, contributions to the TEP due to magnetic, morphological differences, etc. in the microstructure are considered negligible.

Figure 2.a shows changes in the TEP of the reference specimen $(\Delta S)$ due to the austenitization temperature in the steel. Moreover, Fig. 2.b shows the amount of carbides still undissolved at the different heating temperatures. The effect of austenitization temperature on the PAGS in the studied steel is shown in Fig. 2.c. It is important to mention that austenite grain growth affects the TEP through the reduction of grain boundaries that may influence the electrical transport properties of the steel [13]. Likewise, the effect of the austenitization temperature on the volume fraction of retained austenite is presented together with the corresponding hardness values in Fig. 2.d.

Any variation between the TEP value of the reference level and the TEP value at any other austenitization state might show quantitative differences in the austenite grain size and in the volume fractions of $\mathrm{M}_{23} \mathrm{C}_{6}$ carbides and retained austenite in the microstructure. At temperatures higher than $1168{ }^{\circ} \mathrm{C}$, temperature at which carbide dissolution process has finished, only the austenite grain growth and the differences in retained austenite volume fraction can explain the change in the TEP at those temperatures. However, from those results it is not possible to evaluate separately the different contributions that affect the TEP.

In this sense, the TEP measurements at different austenitization temperatures had been carried out in medium carbon manganese steel in which no dissolution process takes place during heating. The chemical composition of this steel in wt-\% is as follows: $0.37 \% \mathrm{C}, 0.72 \% \mathrm{Mn}$ and $0.27 \% \mathrm{Si}$. TEP 
samples were austenitized in vacuum at different temperatures (1000, 1100, 1150, 1200 and 1250 $\left.{ }^{\circ} \mathrm{C}\right)$. After 180 seconds of holding time, specimens were gas quenched. The TEP value corresponding to the lowest austenitization temperature tested $\left(1000^{\circ} \mathrm{C}\right)$, equal to $5.806 \mu \mathrm{V} / \mathrm{K}$, was chosen as the reference level. Fig. 3.a shows the TEP variation and austenite grain diameter of samples quenched from different austenitization temperatures. Since carbide dissolution does not take place during heating, TEP variation is due to the austenite grain growth. Fig. 3.a suggests that the grain boundary affects the electrical transport properties of the steel, but this influence is less significant the smaller the grain boundary surface area in the steel i.e. the higher the austenite grain size. Thus, Fig. 3.b represents the influence of austenite grain growth on the TEP measurements. Points in this graph correspond to experimental values for the studied medium carbon manganese steel, whereas the solid line corresponds to an exponential fitting function that reproduces the influence of austenite grain growth on the TEP measurements in steels. This function takes the following form:

$$
\Delta S_{G B}=1.6\left[1-\exp \left(-\frac{\Delta D}{20}\right)\right]
$$

being $\Delta S_{G B}$ the variation in the TEP measurement due to austenite grain growth, and $\Delta D$ the variation in austenite grain diameter.

Regarding the TEP measurements in the stainless steel, at temperatures higher than $1168{ }^{\circ} \mathrm{C}$ only the austenite grain growth and the differences in retained austenite volume fraction justified the change in the TEP at those temperatures. Once the influence of austenite grain growth on the TEP measurements in the steel is known making use of equation (2), it is possible to evaluate the contribution due to the difference in retained austenite volume fraction that affects the TEP measurement:

$$
\Delta S_{\text {microstr }}=\Delta S^{T \geq 1168^{\circ} \mathrm{C}}-\Delta S_{G B}^{T \geq 1168^{\circ} \mathrm{C}}
$$

where $\Delta S_{\text {microstr }}$ is the variation in the TEP due to the difference in retained austenite volume fraction in the martensite plus retained austenite microstructure, $\Delta S^{T \geq 1168^{\circ} \mathrm{C}}$ is the change in the TEP at temperatures higher than $1168{ }^{\circ} \mathrm{C}$ (Fig. 2.a), and $\Delta S_{G B}^{T \geq 1168^{\circ} \mathrm{C}}$ is the variation in the TEP due to austenite grain growth at the same temperatures estimated from data in Fig. 2.c and equation (2). Figure 4.a represents the influence of microstructure on the TEP measurements in the stainless steel at temperatures higher than $1168{ }^{\circ} \mathrm{C}$ obtained using equation (3). This figure suggests that the presence of austenite to the detriment of martensite in the microstructure increases the TEP. This behavior is explained by the decrease in strain of the microstructure that occurs when the volume fraction of martensite is reduced. It is well known that the reduction of dislocations leads to a positive TEP variation [14].

Assuming that the TEP variation due to the difference in microstructure is proportional to the retained austenite volume fraction, the following function is proposed:

$$
\Delta S_{\text {microstr }}=K_{\text {microstr }} \cdot\left(\Delta \% \gamma_{\text {ret }}\right)
$$

being $K_{\text {microstr }}=0.087 \mu \mathrm{V} / \mathrm{K}$ and $\Delta \% \gamma_{\text {ret }}$ the change in retained austenite volume fraction.

On the other hand, coarse precipitates such as $\mathrm{M}_{23} \mathrm{C}_{6}$ carbides are unlikely to have an intrinsic effect on the TEP. In the studied steel, carbides are $0.9 \mu \mathrm{m}$ in diameter in the as-received microstructure [8]. During the dissolution process of those carbides, the measured TEP variations are essentially related to the increase in solid solution content of elements, chromium and carbon in this work. Fig. 4.b represents the change in the TEP due to the chromium content of Fe-Cr-Ni alloys with a nickel 
content of 5 wt-\% according to Kawaguchi and Yamanaka [15]. According to these results, for chromium contents lower than $20 \mathrm{wt} \%$ the TEP variation associated with chromium in solid solution in the austenite is proportional to the chromium content. Bearing in mind that the chromium content of the studied steel (13 wt-\%) is lower than this value, the following function is proposed to estimate the contribution to the TEP due to the chromium content in solid solution in the austenite:

$$
\Delta S_{C r}^{S S}=K_{C r} \cdot[C r]_{S S}
$$

where $\Delta S_{C r}^{S S}$ is the contribution to the TEP due to the chromium content in solid solution in the austenite, $K_{C r}=-0.30 \mu \mathrm{V} / \mathrm{K}(\mathrm{wt}-\%)$ and $[\mathrm{Cr}]_{S S}$ is the chromium content in wt-\%. This result shows that the effect of chromium in solid solution on the TEP is an order of magnitude lower than that of manganese $\left(K_{M n}=-3 \mu \mathrm{V} / \mathrm{K}(\mathrm{wt}-\%)\right.$ and two orders of magnitude lower than that of aluminum $\left(K_{A l}=-\right.$ $30 \mu \mathrm{V} / \mathrm{K}(\mathrm{wt}-\%)$ [16]. Bearing in mind the atomic size of those elements relative to that of iron $\left(r_{F e}=1.26 \AA, r_{C r}=1.30 \AA, r_{M n}=1.35 \AA\right.$, and $r_{A l}=1.43 \AA$ ), it seems that substitutional atoms with a size similar to that of iron have a lower influence on the TEP. This is a reasonable deduction since substitutional elements with atomic size closer to that of iron cause less distortion in the crystalline structure and in the electron conduction band.

Once the influence of grain boundaries, microstructure and chromium content in solid solution on the TEP measurements has been identified, it is possible to evaluate the contribution due to the carbon content in solid solution in the austenite, $\Delta S_{C}^{S S}$ :

$$
\Delta S_{C}^{S S}=\Delta S-\Delta S_{G B}-\Delta S_{\text {microstr }}-\Delta S_{C r}^{S S}
$$

where $\Delta S$ is the change in the TEP represented in Fig. 2.a. Figure 4.c shows the influence of carbon content in solid solution in the austenite on the TEP measurements for the stainless steel. Points in this graph are obtained using equations (2), (4-6) and considering the amount of chromium and carbon in $\mathrm{M}_{23} \mathrm{C}_{6}$ carbides about 55 wt-\% and 5.4 wt-\%, respectively at all the temperatures tested. Those values have been obtained by thermodynamic calculations [17]. As expected, the TEP measurement decreases as the carbon content in the austenite increases. Assuming that the TEP variation is proportional to the carbon content in solid solution in the austenite, the following function is proposed to estimate the contribution to the TEP due to this element:

$$
\Delta S_{C}^{S S}=K_{C} \cdot[C]_{S S}
$$

being $K_{C}=-20 \mu \mathrm{V} / \mathrm{K}(\mathrm{wt}-\%)$ and $[C]_{S S}$ is the carbon content in solid solution in wt-\%. The results show that the effect of carbon in solid solution on the TEP is much higher than that of chromium. However, the influence of carbon in solid solution on the TEP, determined in the studied stainless steel, is slightly lower than that in low or ultra low carbon steels $\left(K_{C}=-45 \mu \mathrm{V} / \mathrm{K}(\mathrm{wt}-\%)\right)$ [3]. It seems that carbon atoms in high-alloyed steels are less effective as scattering centres for electrons than those in low-alloyed steels.

Figure 5 represents separately the different contributions that affect the TEP during the dissolution processes of $\mathrm{M}_{23} \mathrm{C}_{6}$ carbides in the X45Cr13 stainless steel. This figure suggests that TEP variations in the studied steel are mainly related to the increase in solid solution content of carbon.

\section{Conclusions}


The influence of carbon and chromium contents in solid solution in the austenite, grain boundaries and the microstructure on the TEP measurement during the dissolution of $\mathrm{M}_{23} \mathrm{C}_{6}$ carbides in a X45Cr13 stainless steel have been evaluated. It has been found that TEP variations in the studied steel are mainly related to the increase in solid solution content of carbon. An increase in the prior austenite grain size slightly raises the TEP values. Likewise, the presence of austenite at the expense of martensite in the microstructure increases the TEP. This behavior is related to the decrease in strain of the microstructure that occurs when the volume fraction of martensite is reduced. On the other hand, the effect of chromium on the TEP is less significant than that of carbon, manganese and aluminum. However, the influence of carbon in solid solution on the TEP determined in the studied stainless steel is slightly lower than that determined in low or ultra low carbon steels.

\section{Acknowledgements}

The authors acknowledge financial support from Spanish Ministerio de Ciencia y Tecnología (MAT2000-0412-P4-02). F.G. Caballero would like to thank Spanish Ministerio de Ciencia y Tecnología for the financial support in the form of a Ramón y Cajal contract (Programa RyC 2002).

\section{References}

1. Ion JC, Moisio T, Pedersen TF, Sorensen B, Hansson CM. J. Mater. Sci. 1991; 26: 43.

2. Meng F, Tagashira K, Azuma R, Sohma H. ISIJ Int. 1994; 34: 205.

3. Benkirat D, Merle P, Borrelly R. Acta Metallurgica 1988; 36: 613.

4. Brahmi A, Borrelly R. Scripta Metallurgica et Materialia 1995; 32: 365.

5. Brahmi A, Borrelly R. Acta Metallurgica 1997; 45: 1889.

6. $\quad$ Lavaire N, Merlin J, Sardoy V. Scripta Materialia 2001; 44: 553.

7. Ney Jose Luiggi A. Metallurgical and Materials Transactions A 1998; 29A: 2669.

8. Caballero FG, Álvarez LF, Capdevila C, García de Andrés C. Scripta Materialia 2003; 49: 315.

9. Garcia de Andrés C, Jiménez JA, Álvarez LF. Metallurgical and Materials Transactions A 1996; 27A: 1799.

10. Garcia de Andrés C, Caruana G, Álvarez LF. Materials Science and Engineering 1998; A241: 211.

11. Durnin J, Ridal KA. Journal of the Iron and Steel Institute 1968; 206: 60.

12. Underwood EE. Quantitative Stereology. Addison-Wesley Reading (MA), 1970.

13. Jácome CE, Flórez JM, Gordillo G. Thin Solid Films 2001; 396: 255.

14. Guetaz V, Merlin VMJ, Ravaine D, Soler M. Steel Research 2001; 72: 245.

15. Kawaguchi Y, Yamanaka S. Journal of Alloys and Compounds 2002; 336: 301.

16. Carabajar S, Merlin J, Massardier V, Chabanet S. Materials Science and Engineering 2000; A218: 132.

17. MTDATA: Phase diagram calculation software. Teddington: National Physical Laboratory. 2003. 


\section{Figure Captions}

Figure 1.- Optical micrographs of the steel $0.45 \% \mathrm{C}-13.0 \% \mathrm{Cr}-0.38 \% \mathrm{Ni}-0.32 \% \mathrm{Si}-0.44 \% \mathrm{Mn}-0.03$ $\% \mathrm{P}-0.016 \% \mathrm{~S}$ (wt-\%): a) in as-received condition; after heat treatment at b) $\left.1010{ }^{\circ} \mathrm{C}, \mathrm{c}\right) 1060{ }^{\circ} \mathrm{C}$, d) $1106{ }^{\circ} \mathrm{C}$, e) $1120^{\circ} \mathrm{C}$, f) $1168{ }^{\circ} \mathrm{C}$, g) $1200^{\circ} \mathrm{C}$ and h) $1232^{\circ} \mathrm{C}$. Etched with Murakami's reagent.

Figure 2.- a) TEP variation; b) volume fraction of carbides; c) austenite grain diameter; d) volume fraction of retained austenite and hardness of samples quenched from different austenitization temperatures. Steel with same composition as in Fig. 1.

Figure 3.- a) TEP variation and austenite grain diameter of samples quenched from different austenitization temperatures in a medium carbon manganese steel; b) Influence of austenite grain growth on the TEP measurements.

Figure 4.- a) Influence of microstructure on the TEP measurements; b) TEP variation in Fe-Cr-Ni alloys due to the chromium content [15]; c) Influence of solid solution carbon content in the austenite on the TEP measurements.

Figure 5.- Different contributions that affect the TEP measurement during the dissolution processes of $\mathrm{M}_{23} \mathrm{C}_{6}$ carbides in the $\mathrm{X} 45 \mathrm{Cr} 13$ stainless steel. 


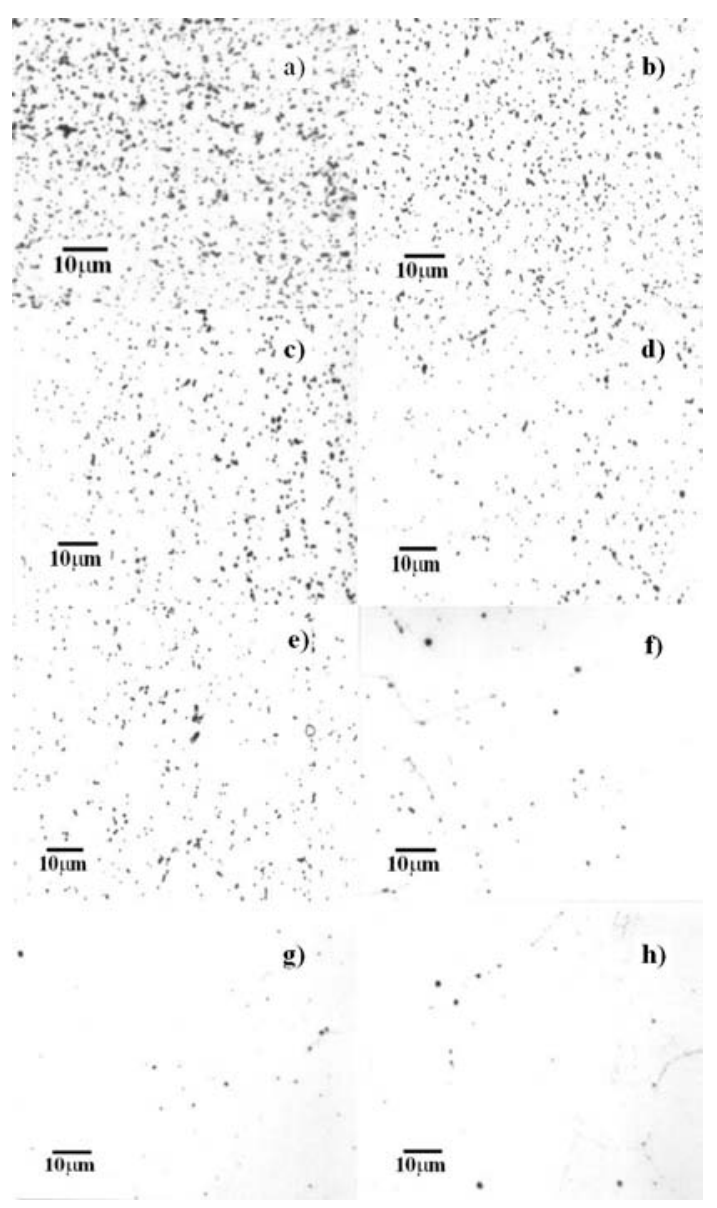

Figure 1 

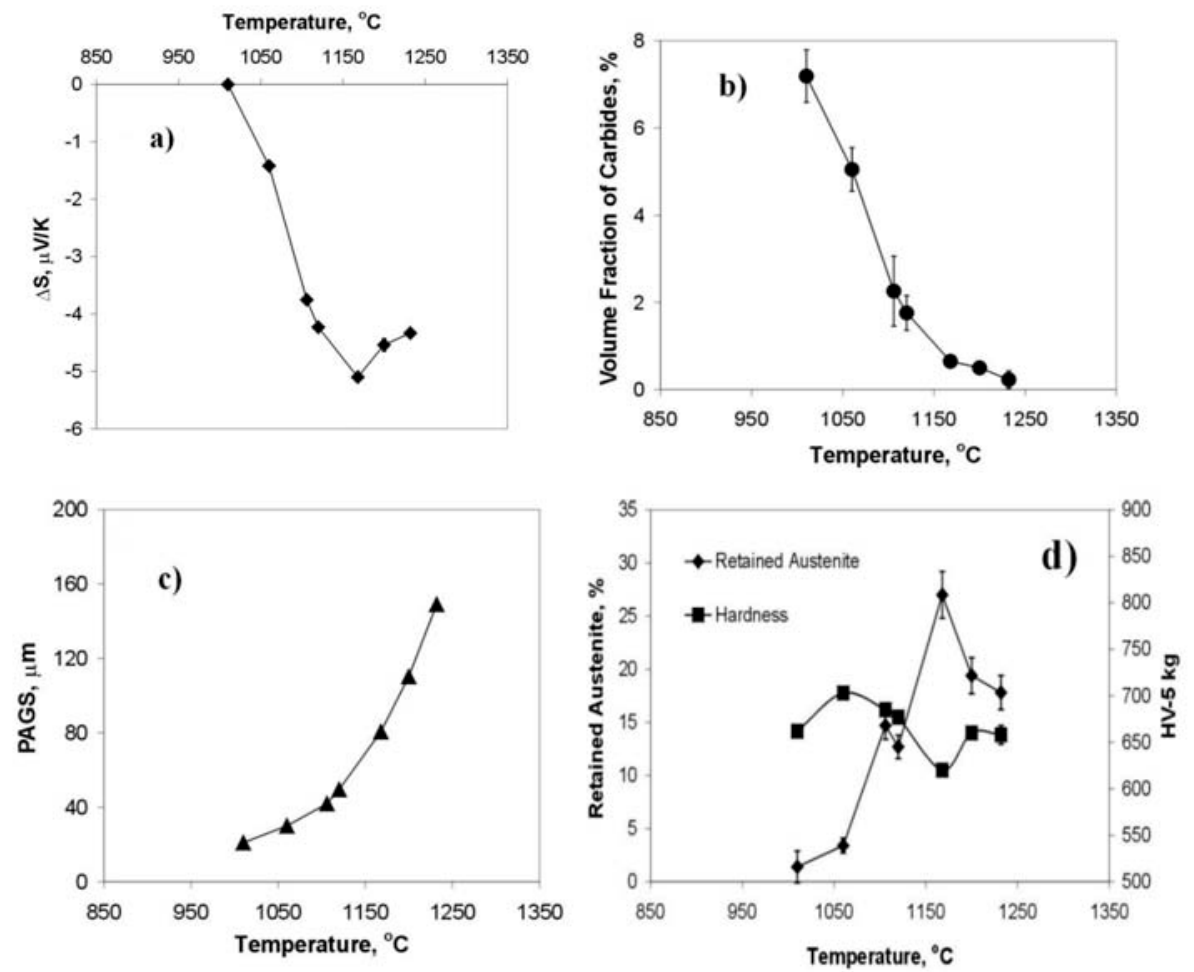

Figure 2 



Figure 3 


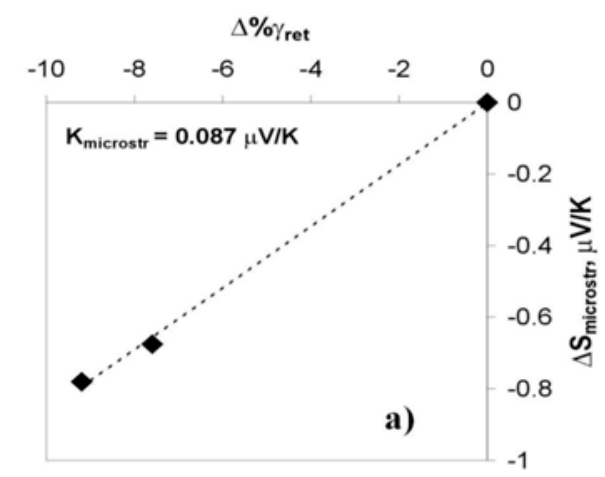

[Cr] $]_{\text {ss, }}$ wt-\%
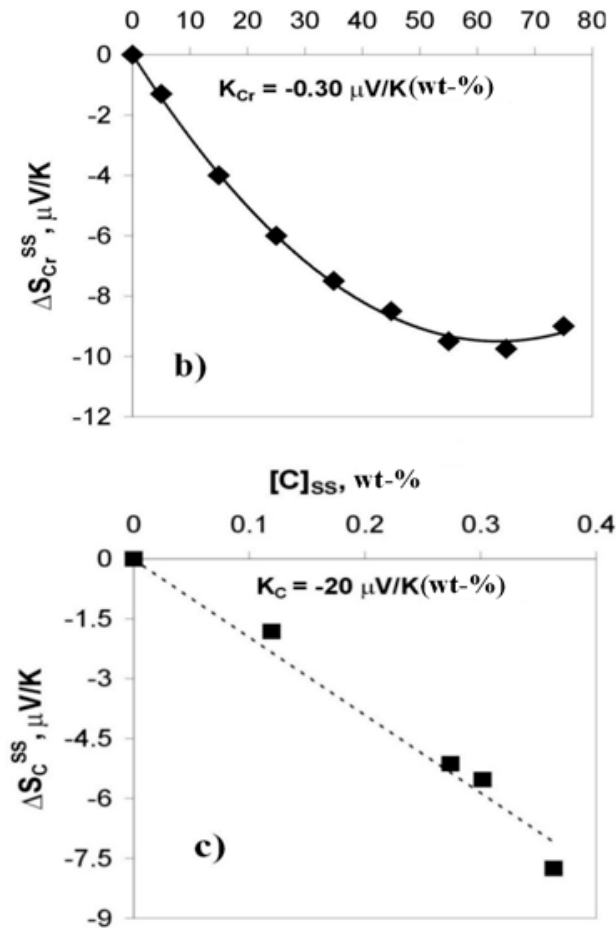

Figure 4 


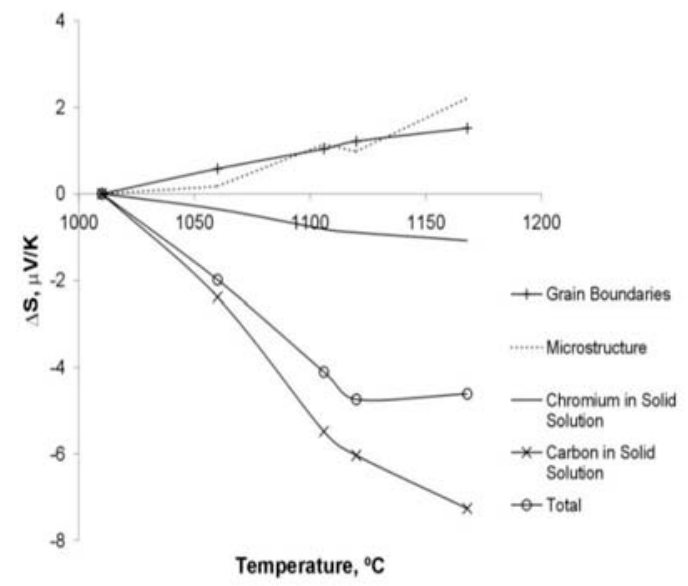

Figure 5 